\title{
Primary Intrapulmonary Thymoma Appearing as a Solitary Pulmonary Nodule: The "Master of Disguise" of Lung Tumors?: Case Report
}

\author{
Athanasios Krassas, M.D., FECTS. ${ }^{1}$, loannis Diamantis, M.D. ${ }^{1}$, loannis Karampinis, M.Sc. ${ }^{1}$, Stefani Vgenopoulou, \\ M.D. ${ }^{2}$, Panagiotis Misthos, M.D., Ph.D. ${ }^{1}$
}

Departments of 'Thoracic Surgery and 'Pathology, Sismanogleio General Hospital, Athens, Greece

\author{
ARTICLE INFO \\ Received September 21, 2020 \\ Revised October 20, 2020 \\ Accepted November 9, 2020 \\ Corresponding author \\ Athanasios Krassas \\ Tel 30-2132058255 \\ Fax 30-2132058255 \\ E-mail athanasekrassas@hotmail.fr \\ ORCID \\ https://orcid.org/0000-0001-5873-5008
}

\begin{abstract}
Primary intrapulmonary thymomas (PITs) are defined as thymomas arising in intrapulmonary locations, without an associated mediastinal component. They are rare lesions, the diagnosis of which can be very difficult. We present a case of PIT in an asymptomatic 74-year-old woman in whom pulmonary nodules were found on pulmonary angiography performed for an episode of pulmonary embolism. She underwent wedge resection and the pathology report revealed a PIT. We also summarize this patient's clinicopathological features and discuss the diagnosis, pathogenesis, and treatment of PIT.
\end{abstract}

Keywords: Thymoma, Pulmonary nodule, Multiple, Diagnosis, Histology, Case report

\section{Case report}

Thymomas originate from thymic epithelial cells, and their incidence is $0.15 / 100,000$ [1]. The vast majority of thymomas occur in the antero-superior mediastinum, but ectopic thymic tissue can be found in the neck, the trachea, the thyroid and parathyroid gland, the pericardium, the heart, the pleura, and the lung. McBurney et al. [2] were the first to report a case of PIT in 1951. Primary intrapulmonary thymomas (PITs) are very rare, and fewer than 50 cases have been reported in the literature. They are defined as tumors with the histopathological characteristics of thymomas, located either as subpleural lesions or surrounded by pulmonary parenchyma without evidence of a lesion in the antero-superior mediastinum. We report a case of PIT in a 74-year-old woman in whom pulmonary nodules were found incidentally.

A 74-year-old woman was admitted to our department due to multiple bilateral pulmonary nodules, which were found incidentally during pulmonary angiography performed for an episode of pulmonary embolism 5 years ago. They were located in the lingula (2 lesions) and in the right upper lobe (2 lesions, measuring $6 \mathrm{~mm}$ ). According to her treating pulmonologist, she was followed up for the nodules, and on repeated chest computed tomography (CT) scans, a lingular lesion increased in size (from 9 to $25 \mathrm{~mm}$ ) during this period, while the other nodules remained stable. She did not present with symptoms of myasthenia gravis or Good syndrome. The mediastinum was normal. She underwent 18-fluorodeoxyglucose positron emission tomography (18FDG-PET)-CT scanning, which showed mild uptake of the lingular nodule (maximum standardized uptake value $\left.\left[\mathrm{SUV}_{\max }\right]=3.4\right)$ without mediastinal involvement. The other sub-centimetric nodules were negative on PETCT (Fig. 1). She underwent wedge resection of the lingular lesion and the lesion in the left upper lobe. The frozen sections of both nodules were negative for malignancy. A macroscopic examination showed a well-circumscribed, encapsulated solid tumor measuring $2 \mathrm{~cm}$ in diameter (Fig. 2). A microscopic examination revealed a cellular neoplastic population of bland ovoid to spindled epithelioid cells with rare, scattered $\mathrm{CDla}^{+}$immature $\mathrm{T}$ lymphocytes. The epithelioid neoplastic cells, following immunohistochemical investigation, stained positive for cytokeratin (CK AE1/ AE3) and p63 and negative for CK7, TTF-1, synaptophysin, chromogranin, CD56, CD117, CD34, CD20, CD5, smooth 


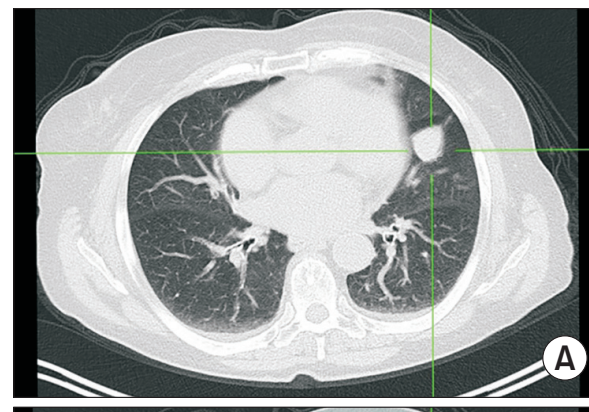

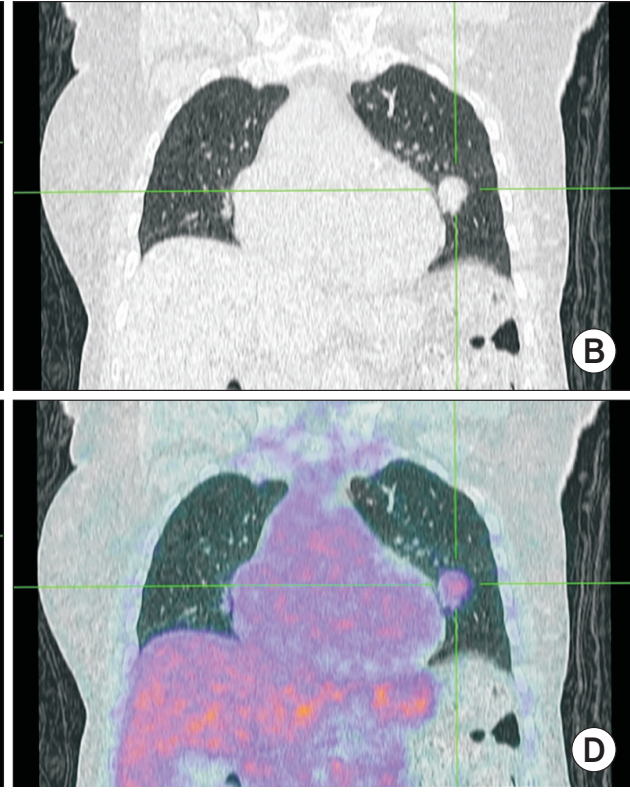

Fig. 1. (A-D) 18-fluorodeoxyglucose positron emission tomography-computed tomography showing a lingular nodule measuring $25 \mathrm{~mm}$ in diameter with mild hypermetabolism (maximum standardized uptake value=3.4).

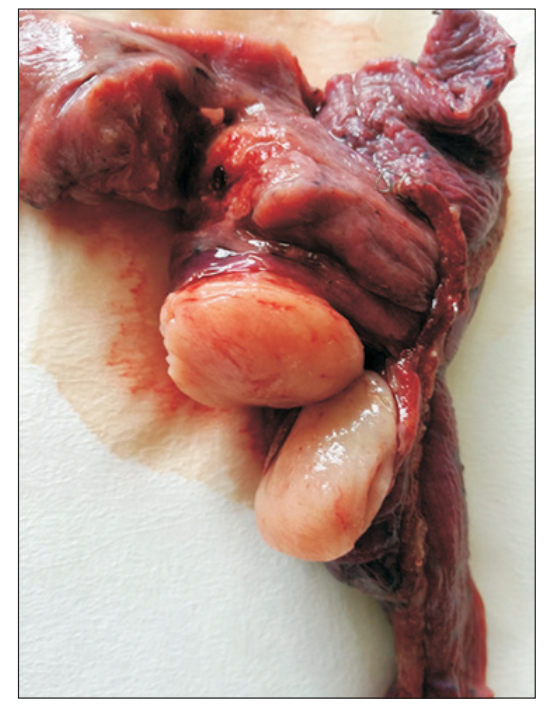

Fig. 2. Specimen of wedge resection of the lingula with a peripheral, well-circumscribed, encapsulated solid tumor $2 \mathrm{~cm}$ in diameter.

muscle actin, desmin, caldesmon, estrogen receptor, progesterone receptor, vimentin, and CD99. The differential diagnosis, based on the microscopic characteristics of the tumor cells, included intrapulmonary thymoma, solitary fibrous tumor, and carcinoid tumor (Fig. 3).

The macroscopic, microscopic, and immunohistochemical findings led to the diagnosis of intrapulmonary thymoma, type A. The left upper lobe nodule was a granuloma. The patient's postoperative course was uneventful, and she was discharged home on the seventh postoperative day.
Based on an oncologic consultation, no adjuvant treatment was administered due to the absence of invasion. It was also decided to monitor the other nodules for potential resection in case of growth. The patient provided written informed consent for the publication of her clinical details and images.

\section{Discussion}

PIT is a rare intrapulmonary tumor, of which fewer than 50 cases have been reported in the literature. PITs are defined as tumors of thymic origin with the characteristic histological features of thymoma, situated below the visceral pleura or completely surrounded by lung parenchyma, without a mediastinal component. The first case was reported by McBurney et al. [2] in 1951. They can be solitary or multiple and they affect both sexes. They usually occur after the fifth decade of life, with a mean age of 57 years. PITs are usually asymptomatic and they are incidentally found on CT scans performed for other reasons. They produce symptoms when they grow large enough to compress or invade neighboring anatomical structures such as the bronchi, parietal pleura, and bronchial arteries, thereby producing pain, cough, bronchial obstruction, and hemoptysis. In addition, they can be associated with paraneoplastic syndromes (in the same way as mediastinal thymomas). The most common of these are myasthenia gravis and Good syndrome.

The pathogenesis of PIT is unknown. The most probable scenario accounting for PIT is that of embryologic rem- 

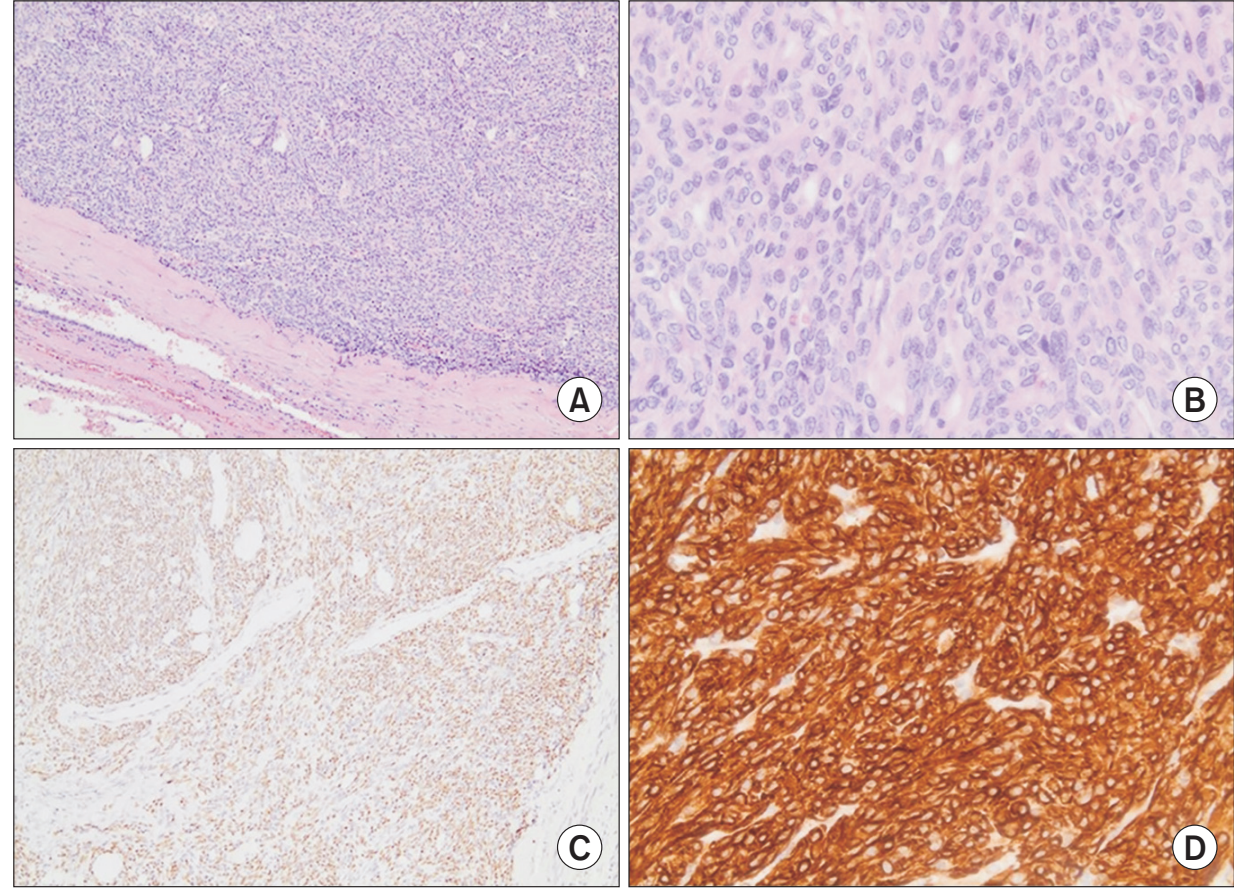

Fig. 3. (A) Cellular intrapulmonary thymoma surrounded by a thick fibrous capsule $(\mathrm{H} \& \mathrm{E}, \times 100)$. (B) Neoplastic epithelioid cells with ovoid spindle cell morphology and lacking significant nuclear atypia $(\mathrm{H} \& \mathrm{E}$, $\times 400$ ). (C) Immunohistochemical staining with p63 antibody showed strong nuclear positivity of the neoplastic cells for this marker (immunohistochemical stain, $\times 100$ ). (D) Strong cytoplasmic positivity of the tumor cells for broad spectrum keratin CKAE1/AE3 (immunohistochemical stain, $\times 200$ ). nants. Normally, the thymus develops from a ventral sacculation of the third pharyngeal pouch, and to a minor extent the fourth pharyngeal pouch, in the sixth week of gestation. During the eighth week, the thymic primordia enlarge towards their lower poles, forming 2 epithelial bars that fuse in the midline, moving caudally to reach the definitive position in the antero-superior mediastinum [3]. During the descent, the tail portion breaks in small fragments that normally disappear. Occasionally, some remnants may persist, producing ectopic thymic tissue and thymomas (located in the neck, thyroid and parathyroid glands, the pleura and the trachea) [4]. Another hypothesis is that PIT arises from stem cells. These cells can differentiate along a variety of lines, thereby producing ectopic mature tissues in the lung parenchyma (thyroid, pancreatic adrenal tissue, etc.). Finally, Fukayama et al. [5] suggested that PIT is the result of a monodermal teratoma.

PIT appears on CT as a well-circumscribed, heterogeneous mass [6]. Our patient also presented with a cylindrical mass with smooth borders. The radiological differential diagnosis of PIT includes low-grade malignant tumor, metastatic lung tumor, hamartoma, sclerosing hemangioma, lipoma, and angiolipoma. PET-CT can be used for the preoperative workup, as for all solitary pulmonary nodules. The degree of 18FDG uptake seems to be related with the histologic subtype of the World Health Organization classification of epithelial tumors. Ishibashi et al. [7] men- tioned that PIT shows mild or no hypermetabolism of 18FDG. In our case, the $\mathrm{SUV}_{\max }$ was 3.4.

Preoperative histologic determination is difficult, and most PITs are diagnosed postoperatively. The diagnosis of PITs is particularly challenging on both biopsy specimens and intraoperative frozen sections, so PIT can be misdiagnosed. To differentiate a PIT from another intrathoracic tumor, the pathologist must rely on immunohistochemical staining combined with the gross and microscopic patterns of the tumor and the patient's clinical history. Macroscopically, the examination shows a well-circumscribed and encapsulated nodule within the pulmonary parenchyma. PITs usually do not involve the visceral pleura. As with mediastinal thymomas, extension beyond the capsule suggests invasiveness, implying a poor prognosis.

Due to the scarcity of available data due to the small number of reported cases, it is difficult to define the optimal treatment strategy of PIT, and radical resection remains the treatment of choice [3]. Depending on the size and the location of the lesion, the extent of resection ranges from wedge resection to pneumonectomy according to the largest review to date, authored by Myers et al. [4]. In case of incomplete resection, adjuvant radiotherapy should be considered. This treatment strategy is based in several retrospective studies of incompletely resected mediastinal thymomas. These studies showed reduced local relapse after radiotherapy. The follow-up period must be relatively 
long, given the substantial risk of recurrence.

In conclusion, PITs are exceedingly rare lesions. Their pathogenesis is currently not clearly understood. Complete resection appears sufficient in non-malignant tumors. In patients who undergo incomplete resection, adjuvant radiotherapy should be considered. Long-term follow-up is warranted because of the risk of late local relapse.

\section{Conflict of interest}

No potential conflict of interest relevant to this article was reported.

\section{ORCID}

Athanasios Krassas: https://orcid.org/0000-0001-5873-5008 Ioannis Diamantis: https://orcid.org/0000-0003-3207-5165 Ioannis Karampinis: https://orcid.org/0000-0003-4363-7047 Stefani Vgenopoulou: https://orcid.org/0000-0002-1454-8814 Panagiotis Misthos: https://orcid.org/0000-0003-2963-1503

\section{References}

1. Engels EA, Pfeiffer RM. Malignant thymoma in the United States: demographic patterns in incidence and associations with subsequent malignancies. Int J Cancer 2003;105:546-51.

2. McBurney RP, Clagett OT, McDonald JR. Primary intrapulmonary neoplasm (thymoma?) associated with myasthenia gravis: report of case. Proc Staff Meet Mayo Clin 1951;26:345-53.

3. Moran CA, Suster S, Fishback NF, Koss MN. Primary intrapulmonary thymoma: a clinicopathologic and immunohistochemical study of eight cases. Am J Surg Pathol 1995;19:304-12.

4. Myers PO, Kritikos N, Bongiovanni M, et al. Primary intrapulmonary thymoma: a systematic review. Eur J Surg Oncol 2007;33:113741.

5. Fukayama M, Maeda Y, Funata N, et al. Pulmonary and pleural thymoma: diagnostic application of lymphocyte markers to the thymoma of unusual site. Am J Clin Pathol 1988;89:617-21.

6. Jung W, Kang CH, Kim YT, Park IK. Primary intrapulmonary thymoma presenting as a solitary pulmonary nodule. Korean J Thorac Cardiovasc Surg 2017;50:54-8.

7. Ishibashi F, Moriya Y, Tamura H, Matsui Y, Iizasa T. Differential diagnosis of primary intrapulmonary thymoma: a report of two cases. Surg Case Rep 2015;1:56. 